\title{
Inclusion and neighborhood properties of certain subclasses of $p$-valent functions of complex order defined by convolution
}

\begin{abstract}
In this paper we introduce and investigate three new subclasses of $p$-valent analytic functions by using the linear operator $D_{\lambda, p}^{m}(f * g)(z)$. The various results obtained here for each of these function classes include coefficient bounds, distortion inequalities and associated inclusion relations for $(n, \theta)$-neighborhoods of subclasses of analytic and multivalent functions with negative coefficients, which are defined by means of a non-homogenous differential equation.
\end{abstract}

1. Introduction. Let $A_{p}(n)$ denote the class of functions of the form

$$
f(z)=z^{p}+\sum_{k=n}^{\infty} a_{k} z^{k} \quad(n>p ; p, n \in \mathbb{N}=\{1,2, \ldots\}),
$$

which are analytic and $p$-valent in the open unit disk $U=\{z:|z|<1\}$. The Hadamard product (or convolution) of the functions $f(z)$ given by (1.1), and $g(z) \in A_{p}(n)$ given by

$$
g(z)=z^{p}+\sum_{k=n}^{\infty} b_{k} z^{k} \quad(n>p ; p, n \in \mathbb{N})
$$

2000 Mathematics Subject Classification. 30C45.

Key words and phrases. Analytic, $p$-valent, $(n, \theta)$-neighborhood, inclusion relations. 
is defined by

$$
(f * g)(z)=z^{p}+\sum_{k=n}^{\infty} a_{k} b_{k} z^{k}=(g * f)(z) .
$$

For functions $f, g \in A_{p}(n)$, we define the linear operator $D_{\lambda, p}^{m}: A_{p}(n) \rightarrow$ $A_{p}(n)\left(\lambda \geq 0 ; p, n \in \mathbb{N} ; m \in \mathbb{N}_{0}=\mathbb{N} \cup\{0\}\right)$ by

$$
\begin{aligned}
D_{\lambda, p}^{0}(f * g)(z) & =(f * g)(z), \\
D_{\lambda, p}^{1}(f * g)(z)=D_{\lambda, p}(f * g)(z) & =(1-\lambda)(f * g)(z)+\frac{\lambda z}{p}(f * g)^{\prime}(z)
\end{aligned}
$$

and (in general)

$$
\begin{aligned}
D_{\lambda, p}^{m}(f * g)(z) & =D_{\lambda, p}\left(D_{\lambda, p}^{m-1}(f * g)(z)\right) \\
& =(1-\lambda) D_{\lambda, p}^{m-1}(f * g)(z)+\frac{\lambda z}{p}\left(D_{\lambda, p}^{m-1}(f * g)\right)^{\prime}(z) \\
& =z^{p}+\sum_{k=n}^{\infty}\left[\frac{p+\lambda(k-p)}{p}\right]^{m} a_{k} b_{k} z^{k}
\end{aligned}
$$

$\left(\lambda \geq 0 ; p, n \in \mathbb{N} ; m \in \mathbb{N}_{0} ; z \in U\right)$.

The operator $D_{\lambda, 1}^{m}(f * g)(z)=D_{\lambda}^{m}(f * g)(z)$ was introduced by Aouf and Seoudy [6].

We note that

(i) for $\lambda=1$ and $b_{k}=1$ (or $\left.g(z)=\frac{z^{p}}{1-z}\right), D_{\lambda, p}^{m}(f * g)(z)=D_{p}^{m} f(z)$, where the operator $D_{p}^{m}$ is the $p$-valent Salagean operator introduced and studied by Aouf and Mostafa [5], Kamali and Orhan [11] and Orhan and Kiziltunc [13];

(ii) for $b_{k}=1$ (or $\left.g(z)=\frac{z^{p}}{1-z}\right), D_{\lambda, p}^{m}(f * g)(z)=D_{\lambda, p}^{m} f(z)$, where the operator $D_{\lambda, p}^{m}$ was introduced and studied by El-Ashwah and Aouf [8].

For a function $f(z) \in A_{p}(n)$, we have

$$
\left(D_{\lambda, p}^{m}(f * g)(z)\right)^{(q)}=\delta(p, q) z^{p-q}+\sum_{k=n}^{\infty} \delta(k, q)\left[\frac{p+\lambda(k-p)}{p}\right]^{m} a_{k} b_{k} z^{k-q}
$$

$\left(\lambda \geq 0 ; p, n \in \mathbb{N} ; q, m \in \mathbb{N}_{0} ; p>q ; z \in U\right)$, where

$$
\delta(p, q)= \begin{cases}1, & (q=0), \\ p(p-1) \ldots(p-q+1), & (q \neq 0) .\end{cases}
$$

We denote by $T_{p}(n)$ the subclass of $A_{p}(n)$ consisting of functions of the form

$$
f(z)=z^{p}-\sum_{k=n}^{\infty} a_{k} z^{k} \quad\left(n>p ; a_{k} \geq 0 ; p, n \in \mathbb{N}\right) .
$$


For a given function $g(z) \in A_{p}(n)$ defined by

$$
g(z)=z^{p}+\sum_{k=n}^{\infty} b_{k} z^{k} \quad\left(b_{k}>0 ; n>p ; p, n \in \mathbb{N}\right),
$$

we now introduce a new subclass $C_{\gamma}^{q}(g(z) ; n, m, p, \lambda, \beta, b)$ of the class $T_{p}(n)$ of $p$-valently analytic functions, which consists of functions $f(z) \in T_{p}(n)$ satisfying the inequality

$$
\left|\frac{1}{b}\left\{\frac{z\left(D_{\lambda, p}^{m}(f * g)(z)\right)^{(q+1)}+\gamma z^{2}\left(D_{\lambda, p}^{m}(f * g)(z)\right)^{(q+2)}}{(1-\gamma)\left(D_{\lambda, p}^{m}(f * g)(z)\right)^{(q)}+\gamma z\left(D_{\lambda, p}^{m}(f * g)(z)\right)^{(q+1)}}-(p-q)\right\}\right|<\beta
$$

$\left(\lambda \geq 0 ; p, n \in \mathbb{N} ; q, m \in \mathbb{N}_{0} ; 0 \leq \gamma \leq 1 ; p>q ; 0<\beta \leq 1 ; b \in \mathbb{C}^{*}=\mathbb{C} \backslash\{0\} ;\right.$ $z \in U)$.

We note that

(1) $C_{0}^{q}(g(z) ; n, 0, p, \lambda, 1, b)=S_{g}(p, n, b, q)$

(Prajapat et al. [14]);

(2) $C_{\gamma}^{q}\left(z^{p}+\sum_{k=n+p}^{\infty}\left(\frac{k+\mu}{p+\mu}\right)^{r} z^{k} ; n+p, 0, p, \lambda, 1, b\right)=S_{n, q}^{p}(\mu, r, \gamma, b)$

$\left(\mu \geq 0\right.$ and $\left.r \in \mathbb{N}_{0}\right)$ (Srivastava et al. [18]);

(3) $C_{0}^{q}\left(z^{p}+\sum_{k=n+p}^{\infty}\left[1+\frac{\zeta(k-p)}{p+r}\right]^{\eta} z^{k} ; n+p, 0, p, \lambda, 1, b\right)=H_{n, q}^{p, r}(b, \zeta, \eta)$

$(\zeta, \eta, r \in \mathbb{R} ; \zeta \geq 0, \eta \geq 0, r \geq 0$ ) (Mahzoon and Latha [12]);

(4) $C_{\gamma}^{q}\left(\frac{z^{p}}{1-z} ; n+p, 0, p, \lambda, \beta, b\right)=S_{n, p}^{q}(\gamma, \beta, b)$

(Altintaş et al. [2]);

(5) $C_{0}^{q}\left(z^{p}+\sum_{k=n+p}^{\infty}\left(\begin{array}{c}\mu+k-1 \\ k-p\end{array}\right) z^{k} ; n+p, 0, p, \lambda, 1, b\right)=H_{n, q}^{p}(\mu, b)$

$(\mu \geq 0)$ (Raina and Srivastava [15]);

(6) $C_{\gamma}^{q}\left(\frac{z^{p}}{1-z} ; n+p, 0, p, \lambda, p-q-\alpha, 1\right)$

$$
=C_{\gamma}^{q}\left(\frac{z^{p}}{1-z} ; n+p, 0, p, \lambda, 1, p-q-\alpha\right)=T_{n}(p, q, \alpha, \gamma)
$$

$(0 \leq \alpha<p-q)$ (Altintaş [1]);

(7) $C_{\gamma}^{q}(g(z) ; n, 0, p, \lambda, \beta, b)=C_{\gamma}^{q}(g(z) ; n, p, \beta, b)$

(Srivastava and Orhan [17] and Aouf [4]);

(8) $C_{0}^{0}\left(\frac{z^{p}}{1-z} ; n, m, p, \lambda, \beta, b\right)=T_{n-p}(m, p, \lambda, b, \beta)$

(El-Ashwah and Aouf [8]). 
Also, we note that

$$
\begin{aligned}
& C_{\gamma}^{q}\left(z^{p}+\sum_{k=n}^{\infty}\left[\frac{p+\ell+\zeta(k-p)}{p+\ell}\right]^{s} z^{k} ; n, 0, p, \lambda, \beta, b\right) \\
& =C_{\gamma}^{q}(\zeta, \ell, s ; n, p, \beta, b) \\
& =\left\{f \in T_{p}(n):\left|\frac{1}{b}\left\{\frac{z\left(I_{p}^{s}(\zeta, \ell) f(z)\right)^{(q+1)}+\gamma z^{2}\left(I_{p}^{s}(\zeta, \ell) f(z)\right)^{(q+2)}}{(1-\gamma)\left(I_{p}^{s}(\zeta, \ell) f(z)\right)^{(q)}+\gamma z\left(I_{p}^{s}(\zeta, \ell) f(z)\right)^{(q+1)}}-(p-q)\right\}\right|<\beta,\right. \\
& p, n \in \mathbb{N} ; q, s \in \mathbb{N}_{0} ; 0 \leq \gamma \leq 1 ; p>q ; 0<\beta \leq 1 ; \\
& \left.\ell, \zeta \geq 0 ; b \in \mathbb{C}^{*} ; z \in U\right\},
\end{aligned}
$$

where $I_{p}^{s}(\zeta, \ell)$ is an extended multiplier transformation (see Cătaş [7]), defined by

$$
I_{p}^{s}(\zeta, \ell) f(z)=z^{p}-\sum_{k=n}^{\infty}\left[\frac{p+\ell+\zeta(k-p)}{p+\ell}\right]^{s} a_{k} z^{k}
$$

$\left(\ell, \zeta \geq 0 ; p \in \mathbb{N}\right.$ and $\left.s \in \mathbb{N}_{0}\right)$

$$
\begin{aligned}
& C_{\gamma}^{q}\left(\frac{z^{p}}{1-z} ; n, m, p, \lambda, \beta, b\right) \\
& =C_{\gamma}^{q}(n, m, p, \lambda, \beta, b) \\
& =\left\{f \in T_{p}(n):\left|\frac{1}{b}\left\{\frac{z\left(D_{\lambda, p}^{m} f(z)\right)^{(q+1)}+\gamma z^{2}\left(D_{\lambda, p}^{m} f(z)\right)^{(q+2)}}{(1-\gamma)\left(D_{\lambda, p}^{m} f(z)\right)^{(q)}+\gamma z\left(D_{\lambda, p}^{m} f(z)\right)^{(q+1)}}-(p-q)\right\}\right|<\beta,\right. \\
& p, n \in \mathbb{N} ; q, m \in \mathbb{N}_{0} ; b \in \mathbb{C}^{*} ; 0 \leq \gamma \leq 1 ; p>q ; \\
& 0<\beta \leq 1 ; \lambda \geq 0\} .
\end{aligned}
$$

Also let $R_{\gamma}^{q}(g(z) ; n, m, p, \lambda, \beta, b)$ denote the subclass $T_{p}(n)$ consisting of functions $f(z)$ of the form (1.9) and the function $g(z)$ of the form (1.10) which satisfy the following inequality:

$$
\left|\frac{1}{b}\left\{(1-\gamma) \frac{\left(D_{\lambda, p}^{m}(f * g)(z)\right)^{(q)}}{z^{p-q}}+\gamma \frac{\left(D_{\lambda, p}^{m}(f * g)(z)\right)^{(q+1)}}{(p-q) z^{p-q-1}}-\delta(p, q)\right\}\right|<\beta
$$

$\left(\lambda \geq 0 ; p, n \in \mathbb{N} ; q, m \in \mathbb{N}_{0} ; 0 \leq \gamma \leq 1 ; p>q ; 0<\beta \leq 1 ; b \in \mathbb{C}^{*} ; z \in U\right)$.

In this paper we shall study some properties of the classes $C_{\gamma}^{q}(g(z) ; n, m$, $p, \lambda, \beta, b)$ and $R_{\gamma}^{q}(g(z) ; n, m, p, \lambda, \beta, b)$ and derive several results for functions in the subclass $H_{\gamma}^{q}(g(z) ; n, m, p, \lambda, \beta, b, \alpha)$ of the function class $T_{p}(n)$, which is defined as follows:

A function $f(z) \in T_{p}(n)$ is said to belong to the class $H_{\gamma}^{q}(g(z) ; n, m, p, \lambda$, $\beta, b, \alpha)$ if $w=f(z)$ satisfies the following non-homogenous Cauchy-Euler 
differential equation:

$$
\begin{aligned}
z^{2} \frac{d^{q+2} w}{d z^{q+2}}+2(1+\alpha) z \frac{d^{q+1} w}{d z^{q+1}} & +\alpha(1+\alpha) \frac{d^{q} w}{d z^{q}} \\
& =(p-q+\alpha)(p-q+\alpha+1) \frac{d^{q} k}{d z^{q}},
\end{aligned}
$$

where $k(z) \in C_{\gamma}^{q}(g(z) ; n, m, p, \lambda, \beta, b)$ and $\alpha>q-p, \alpha \in R, p \in \mathbb{N}, q \in \mathbb{N}_{0}$.

2. Basic properties of the classes $C_{\gamma}^{q}(g(z) ; n, m, p, \lambda, \beta, b)$ and $\boldsymbol{R}_{\boldsymbol{\gamma}}^{q}(\boldsymbol{g}(\boldsymbol{z}) ; \boldsymbol{n}, \boldsymbol{m}, \boldsymbol{p}, \boldsymbol{\lambda}, \boldsymbol{\beta}, \boldsymbol{b})$. We begin by proving a necessary and sufficient condition for a function belonging to the class $T_{p}(n)$ to be in the class $C_{\gamma}^{q}(g(z) ; n, m, p, \lambda, \beta, b)$.

Theorem 1. Let the function $f(z) \in T_{p}(n)$ be defined by (1.9) and let $g(z)$ be defined by (1.10). Then $f(z)$ is in the class $C_{\gamma}^{q}(g(z) ; n, m, p, \lambda, \beta, b)$ if and only if

$$
\begin{gathered}
\sum_{k=n}^{\infty}[k-p+\beta|b|][1+\gamma(k-q-1)]\left[\frac{p+\lambda(k-p)}{p}\right]^{m} \delta(k, q) a_{k} b_{k} \\
\leq \beta|b|[1+\gamma(p-q-1)] \delta(p, q) .
\end{gathered}
$$

Proof. If the condition (2.1) holds true, we find from (1.9), (1.10) and (2.1) that

$$
\begin{aligned}
& \mid z\left(D_{\lambda, p}^{m}(f * g)(z)\right)^{(q+1)}+\gamma z^{2}\left(D_{\lambda, p}^{m}(f * g)(z)\right)^{(q+2)} \\
& \quad-(p-q)\left[(1-\gamma)\left(D_{\lambda, p}^{m}(f * g)(z)\right)^{(q)}+\gamma z\left(D_{\lambda, p}^{m}(f * g)(z)\right)^{(q+1)}\right] \mid \\
& \quad-\beta\left|b\left[(1-\gamma)\left(D_{\lambda, p}^{m}(f * g)(z)\right)^{(q)}+\gamma z\left(D_{\lambda, p}^{m}(f * g)(z)\right)^{(q+1)}\right]\right| \\
& =\mid \delta(p, q+1) z^{p-q}-\sum_{k=n}^{\infty}\left[\frac{p+\lambda(k-p)}{p}\right]^{m} \delta(k, q+1) a_{k} b_{k} z^{k-q} \\
& \quad+\gamma \delta(p, q+2) z^{p-q}-\sum_{k=n}^{\infty} \gamma\left[\frac{p+\lambda(k-p)}{p}\right]^{m} \delta(k, q+2) a_{k} b_{k} z^{k-q} \\
& \quad-(p-q)\left[(1-\gamma) \delta(p, q) z^{p-q}-\sum_{k=n}^{\infty}(1-\gamma)\left[\frac{p+\lambda(k-p)}{p}\right]^{m} \delta(k, q) a_{k} b_{k} z^{k-q}\right. \\
& \left.\quad+\gamma \delta(p, q+1) z^{p-q}-\sum_{k=n}^{\infty} \gamma\left[\frac{p+\lambda(k-p)}{p}\right]^{m} \delta(k, q+1) a_{k} b_{k} z^{k-q}\right] \mid \\
& \quad-\beta \mid b\left[(1-\gamma) \delta(p, q) z^{p-q}-\sum_{k=n}^{\infty}(1-\gamma)\left[\frac{p+\lambda(k-p)}{p}\right]^{m} \delta(k, q) a_{k} b_{k} z^{k-q}\right. \\
& \left.\quad+\gamma \delta(p, q+1) z^{p-q}-\sum_{k=n}^{\infty} \gamma\left[\frac{p+\lambda(k-p)}{p}\right]^{m} \delta(k, q+1) a_{k} b_{k} z^{k-q}\right] \mid
\end{aligned}
$$




$$
\begin{aligned}
=\left|\sum_{k=n}^{\infty}(k-p)[1+\gamma(k-q-1)]\left[\frac{p+\lambda(k-p)}{p}\right]^{m} \delta(k, q) a_{k} b_{k} z^{k-q}\right| & \\
& -\beta \mid b\left[(1+\gamma(p-q-1)) \delta(p, q) z^{p-q}\right. \\
& \left.\quad-\sum_{k=n}^{\infty}(1+\gamma(k-q-1))\left[\frac{p+\lambda(k-p)}{p}\right]^{m} \delta(k, q) a_{k} b_{k} z^{k-q}\right] \mid \\
\leq & \sum_{k=n}^{\infty}(k-p)[1+\gamma(k-q-1)]\left[\frac{p+\lambda(k-p)}{p}\right]^{m} \delta(k, q) a_{k} b_{k}|z|^{k-p} \\
& \quad-\beta|b|\{[1+\gamma(p-q-1)] \delta(p, q) \\
& \left.\quad-\sum_{k=n}^{\infty}[1+\gamma(k-q-1)]\left[\frac{p+\lambda(k-p)}{p}\right]^{m} \delta(k, q) a_{k} b_{k}|z|^{k-p}\right\} \\
\leq \sum_{k=n}^{\infty}[k-p+\beta|b|][1+\gamma(k-q-1)]\left[\frac{p+\lambda(k-p)}{p}\right]^{m} \delta(k, q) a_{k} b_{k} & \quad-\beta|b|[1+\gamma(p-q-1)] \delta(p, q) \leq 0
\end{aligned}
$$

$(z \in \partial U=\{z: z \in \mathbb{C}$ and $|z|=1\})$. Hence, by the maximum modulus theorem, $f(z) \in C_{\gamma}^{q}(g(z) ; n, p, \beta, b)$.

Conversely, let $f(z) \in C_{\gamma}^{q}(g(z) ; n, p, \beta, b)$ be given by (1.9) and $g(z)$ be given by (1.10). Then from (1.7) and (1.11), we have

$$
\begin{aligned}
& \left|\frac{1}{b}\left\{\frac{z\left(D_{\lambda, p}^{m}(f * g)(z)\right)^{(q+1)}+\gamma z^{2}\left(D_{\lambda, p}^{m}(f * g)(z)\right)^{(q+2)}}{(1-\gamma)\left(D_{\lambda, p}^{m}(f * g)(z)\right)^{(q)}+\gamma z\left(D_{\lambda, p}^{m}(f * g)(z)\right)^{(q+1)}}-(p-q)\right\}\right| \\
& =\left|\frac{1}{b}\left\{\frac{\sum_{k=n}^{\infty}(k-p)[1+\gamma(k-q-1)]\left[\frac{p+\lambda(k-p)}{p}\right]^{m} \delta(k, q) a_{k} b_{k} z^{k-p}}{[1+\gamma(p-q-1)] \delta(p, q)-\sum_{k=n}^{\infty}[1+\gamma(k-q-1)]\left[\frac{p+\lambda(k-p)}{p}\right]^{m} \delta(k, q) a_{k} b_{k} z^{k-p}}\right\}\right| \\
& <\beta .
\end{aligned}
$$

Putting $z=r(0 \leq r<1)$ on the right-hand side of $(2.2)$ and noting the fact that for $r=0$, the resulting expression in the denominator is positive and remains so for all $r \in(0,1)$, the desired inequality (2.1) follows upon letting $r \rightarrow 1^{-}$.

Theorem 2. Let the function $f(z) \in T_{p}(n)$ be defined by (1.9) and $g(z)$ be defined by (1.10). Then $f(z)$ is in the class $R_{\gamma}^{q}(g(z) ; n, m, p, \lambda, \beta, b)$ if and only if

$$
\sum_{k=n}^{\infty}[p-q+\gamma(k-p)]\left[\frac{p+\lambda(k-p)}{p}\right]^{m} \delta(k, q) a_{k} b_{k} \leq \beta|b|(p-q) .
$$


Corollary 1. Let the function $f(z) \in T_{p}(n)$ be given by (1.9) and $g(z)$ be defined by (1.10). If $f(z) \in C_{\gamma}^{q}(g(z) ; n, m, p, \lambda, \beta, b)$, then

$$
a_{k} \leq \frac{\beta|b|[1+\gamma(p-q-1)] \delta(p, q)}{[k-p+\beta|b|][1+\gamma(k-q-1)]\left[\frac{p+\lambda(k-p)}{p}\right]^{m} \delta(k, q) b_{k}}
$$

$\left(k \geq n ; \lambda \geq 0 ; 0 \leq \gamma \leq 1 ; 0<\beta \leq 1 ; b \in \mathbb{C}^{*} ; p, n \in \mathbb{N} ; q, m \in \mathbb{N}_{0}\right)$.

The result is sharp for the function $f(z)$ given by

$$
f(z)=z^{p}-\frac{\beta|b|[1+\gamma(p-q-1)] \delta(p, q)}{\delta(k, q)[k-p+\beta|b|][1+\gamma(k-q-1)]\left[\frac{p+\lambda(k-p)}{p}\right]^{m} b_{k}} z^{k}
$$

$\left(k \geq n ; \lambda \geq 0 ; 0 \leq \gamma \leq 1 ; 0<\beta \leq 1 ; b \in \mathbb{C}^{*} ; p, n \in \mathbb{N} ; q, m \in \mathbb{N}_{0}\right)$.

We next prove the following growth and distortion property for the functions of the form (1.9) belonging to the class $C_{\gamma}^{q}(g(z) ; n, m, p, \lambda, \beta, b)$.

Theorem 3. If a function $f(z)$ defined by $(1.9)$ is in the class $C_{\gamma}^{q}(g(z) ; n, m$, $p, \lambda, \beta, b)$ and $g(z)$ defined by (1.10). Then

$$
\begin{aligned}
& \left.\quad|| f(z)|-| z\right|^{p} \mid \\
& \quad \leq \frac{\beta|b|[1+\gamma(p-q-1)] \delta(p, q)}{(n-p+\beta|b|)[1+\gamma(n-q-1)]\left[\frac{p+\lambda(n-p)}{p}\right]^{m} \delta(n, q) b_{n}}|z|^{n}
\end{aligned}
$$

$\left(\lambda \geq 0 ; p, n \in \mathbb{N} ; q, m \in \mathbb{N}_{0} ; 0 \leq \gamma \leq 1 ; n>p>q ; 0<\beta \leq 1 ; b \in \mathbb{C}^{*} ;\right.$ $z \in U$ ) and (in general)

$$
\begin{aligned}
& \|\left|f^{(r)}(z)\right|-\delta(p, r)|z|^{p-r} \mid \\
& \quad \leq \frac{\beta|b|[1+\gamma(p-q-1)](n-q) ! \delta(p, q)}{(n-p+\beta|b|)(n-r) ![1+\gamma(n-q-1)]\left[\frac{p+\lambda(n-p)}{p}\right]^{m} b_{n}}|z|^{n-r}
\end{aligned}
$$

$\left(z \in U ; p, n \in \mathbb{N} ; n>p ; m, q \in \mathbb{N}_{0} ; r \leq q<p ; p>\max (r, q) ; \lambda \geq 0\right)$. The result is sharp for the function $f(z)$ given by

$$
f(z)=z^{p}-\frac{\beta|b|[1+\gamma(p-q-1)] \delta(p, q)}{(n-p+\beta|b|)[1+\gamma(n-q-1)]\left[\frac{p+\lambda(n-p)}{p}\right]^{m} \delta(n, q) b_{n}} z^{n}
$$

$(n>p ; p, n \in \mathbb{N})$.

Proof. In view of Theorem 1, we have

$$
\begin{aligned}
& (n-p+\beta|b|)[1+\gamma(n-q-1)]\left[\frac{p+\lambda(n-p)}{p}\right]^{m} \delta(n, q) b_{n} \sum_{k=n}^{\infty} a_{k} \\
& \quad \leq \sum_{k=n}^{\infty}[k-p+\beta|b|][1+\gamma(k-q-1)]\left[\frac{p+\lambda(k-p)}{p}\right]^{m} \delta(k, q) a_{k} b_{k} \\
& \quad \leq \beta|b|[1+\gamma(p-q-1)] \delta(p, q),
\end{aligned}
$$


which readily yields

$$
\sum_{k=n}^{\infty} a_{k} \leq \frac{\beta|b|[1+\gamma(p-q-1)] \delta(p, q)}{(n-p+\beta|b|)[1+\gamma(n-q-1)]\left[\frac{p+\lambda(n-p)}{p}\right]^{m} \delta(n, q) b_{n}} .
$$

Also, (2.1) yields

$$
\sum_{k=n}^{\infty} k ! a_{k} \leq \frac{\beta|b|[1+\gamma(p-q-1)](n-q) ! \delta(p, q)}{(n-p+\beta|b|)[1+\gamma(n-q-1)]\left[\frac{p+\lambda(n-p)}{p}\right]^{m} b_{n}}
$$

Now, by differentiating $r$ times both sides of (1.9), we have

$$
f^{(r)}(z)=\delta(p, r) z^{p-r}-\sum_{k=n}^{\infty} \delta(k, r) a_{k} z^{k-r}
$$

$\left(p, n \in \mathbb{N} ; r \in \mathbb{N}_{0} ; p>r\right)$.

Theorem 3 follows from (2.9), (2.10) and (2.11). Finally, it is easy to see that the bounds in Theorem 1 are attained for the function $f(z)$ given by $(2.8)$.

3. Properties of the class $H_{\gamma}^{q}(g(z) ; n, m, p, \lambda, \beta, b, \alpha)$. Applying the results of Section 2, which are obtained for the function $f(z)$ of the form (1.9) belonging to the class $C_{\gamma}^{q}(g(z) ; n, m, p, \lambda, \beta, b)$, we now derive the corresponding results for the function $f(z)$ belonging to the class $H_{\gamma}^{q}(g(z) ; n, m, p$, $\lambda, \beta, b, \alpha)$.

Theorem 4. If a function $f(z)$ is defined by (1.9) and $g(z)$ is defined by (1.10), and $f(z)$ is in the class $H_{\gamma}^{q}(g(z) ; n, m, p, \lambda, \beta, b, \alpha)$. Then

$$
\begin{aligned}
& \left.|| f(z)|-| z\right|^{p} \mid \\
& \leq \frac{\beta|b|[1+\gamma(p-q-1)](p-q+\alpha)(p-q+\alpha+1) \delta(p, q)}{(n-p+\beta|b|)[1+\gamma(n-q-1)]\left[\frac{p+\lambda(n-p)}{p}\right]^{m}(n-q+\alpha) \delta(n, q) b_{n}}|z|^{n}
\end{aligned}
$$

and (in general)

$$
\begin{aligned}
& \left.|| f^{(r)}(z)|-\delta(p, r)| z\right|^{p-r} \mid \\
& \leq \frac{\beta|b|[1+\gamma(p-q-1)](p-q+\alpha)(p-q+\alpha+1)(n-q) ! \delta(p, q)}{(n-p+\beta|b|)[1+\gamma(n-q-1)]\left[\frac{p+\lambda(n-p)}{p}\right]^{m}(n-q+\alpha)(n-r) ! b_{n}}|z|^{n-r}
\end{aligned}
$$

$\left(p, n \in \mathbb{N} ; m, q \in \mathbb{N}_{0} ; r \leq q<p ; p>\max (r, q) ; 0 \leq \gamma \leq 1 ; 0<\beta \leq 1\right.$; $\left.b \in \mathbb{C}^{*} ; \lambda \geq 0 ; z \in U\right)$. The results in (3.1) and (3.2) are sharp for the function $f(z)$ given by

$$
f(z)=z^{p}-\frac{\beta|b| \delta(p, q)[1+\gamma(p-q-1)](p-q+\alpha)(p-q+\alpha+1)}{(n+\beta|b|) \delta(n+p, q)[1+\gamma(n+p-q-1)](n+p-q+\alpha) b_{n+p}} z^{n}
$$


Proof. Assume that $f(z) \in T_{p}(n)$ is given by (1.9) and $g(z)$ given by (1.10). Also, let function $k(z) \in C_{\gamma}^{q}(g(z) ; n, m, p, \lambda, \beta, b)$, occurring in the non-homogenous differential equation (1.13) be of the form:

$$
k(z)=z^{p}-\sum_{k=n}^{\infty} c_{k} z^{k}
$$

$\left(c_{k} \geq 0 ; n>p ; p, n \in \mathbb{N}\right)$. Then, we readily find from (1.13) that

$$
a_{k}=\frac{(p-q+\alpha)(p-q+\alpha+1)}{(k-q+\alpha)(k-q+\alpha+1)} c_{k}
$$

$(k \geq n ; p, n \in \mathbb{N})$, so that

$$
f(z)=z^{p}-\sum_{k=n}^{\infty} a_{k} z^{k}=z^{p}-\sum_{k=n}^{\infty} \frac{(p-q+\alpha)(p-q+\alpha+1)}{(k-q+\alpha)(k-q+\alpha+1)} c_{k} z^{k}
$$

$(z \in U)$, and

$$
\left.\left.|| f(z)|-| z\right|^{p}|\leq| z\right|^{n} \sum_{k=n}^{\infty} \frac{(p-q+\alpha)(p-q+\alpha+1)}{(k-q+\alpha)(k-q+\alpha+1)} c_{k}
$$

$(z \in U)$. Next, since $k(z) \in C_{\gamma}^{q}(g(z) ; n, m, p, \lambda, \beta, b)$, therefore, on using the assertion (2.4) of Corollary 1 , we get the following coefficient inequality:

$$
c_{k} \leq \frac{\beta|b|[1+\gamma(p-q-1)] \delta(p, q)}{(n-p+\beta|b|)[1+\gamma(n-q-1)]\left[\frac{p+\lambda(n-p)}{p}\right]^{m} \delta(n, q) b_{n}}
$$

$\left(k \geq n ; n>p>q ; \lambda \geq 0 ; 0 \leq \gamma \leq 1 ; 0<\beta \leq 1 ; p, n \in \mathbb{N} ; q, m \in \mathbb{N}_{0}\right.$; $\left.b \in \mathbb{C}^{*}\right)$, which in conjunction with (3.6) and (3.7) yields

$$
\begin{aligned}
& \left.|| f(z)|-| z\right|^{p} \mid \\
& \quad \leq \frac{\beta|b|[1+\gamma(p-q-1)](p-q+\alpha)(p-q+\alpha+1) \delta(p, q)}{(n-p+\beta|b|)[1+\gamma(n-q-1)]\left[\frac{p+\lambda(n-p)}{p}\right]^{m} \delta(n, q) b_{n}}|z|^{n} \\
& \quad \times \sum_{k=n}^{\infty} \frac{1}{(k-q+\alpha)(k-q+\alpha+1)}
\end{aligned}
$$

$(z \in U)$. Note that the following summation result holds

$$
\begin{aligned}
\sum_{k=n}^{\infty} \frac{1}{(k-q+\alpha)(k-q+\alpha+1)} & =\sum_{k=n}^{\infty}\left(\frac{1}{(k-q+\alpha)}-\frac{1}{(k-q+\alpha+1)}\right) \\
& =\frac{1}{(n-q+\alpha)},
\end{aligned}
$$

where $\alpha \in \mathbb{R}^{*}=\mathbb{R} \backslash\{-n,-n-1, \ldots\}$. The assertion (3.1) of Theorem 4 follows from (3.9) and (3.10), respectively. The assertion (3.2) of Theorem 4 
can be established similarly by applying (2.10), (2.11), (3.5) and (3.10), respectively.

4. Inclusion relations involving $(n, \theta)$-neighborhood for the classes $C_{\gamma}^{q}(g(z) ; n, m, p, \lambda, \beta, b), R_{\gamma}^{q}(g(z) ; n, m, p, \lambda, \beta, b)$ and $H_{\gamma}^{q}(g(z) ; n$, $\boldsymbol{m}, \boldsymbol{p}, \boldsymbol{\lambda}, \boldsymbol{\beta}, \boldsymbol{b}, \boldsymbol{\alpha})$. Following the works of Goodman [10], Ruscheweyh [16] and Altintas [1] (see also [2], [3] and [9]), we define the $(n, \theta)$-neighborhood of a function $f^{(q)}(z)$ when $f \in T_{p}(n)$ by

$$
\begin{aligned}
& N_{n, p}^{\theta}\left(f^{(q)}, k^{(q)}\right) \\
& \quad=\left\{k \in T_{p}(n): k(z)=z^{p}-\sum_{k=n}^{\infty} c_{k} z^{k} \text { and } \sum_{k=n}^{\infty} k \delta(k, q)\left|a_{k}-c_{k}\right| \leq \theta\right\} .
\end{aligned}
$$

It follows from (4.1) that, if

$$
h(z)=z^{p}
$$

$(p \in \mathbb{N})$, then

$$
\begin{aligned}
& N_{n, p}^{\theta}\left(h^{(q)}\right) \\
& \quad=\left\{k \in T_{p}(n): k(z)=z^{p}-\sum_{k=n}^{\infty} c_{k} z^{k} \text { and } \sum_{k=n+p}^{\infty} k \delta(k, q)\left|c_{k}\right| \leq \theta\right\} .
\end{aligned}
$$

Next, we establish inclusion relationships for the function classes $C_{\gamma}^{q}(g(z)$; $n, m, p, \lambda, \beta, b)$ and $R_{\gamma}^{q}(g(z) ; n, m, p, \lambda, \beta, b)$, involving the $(n, \theta)$-neighborhood $N_{n, p}^{\theta}\left(h^{(q)}\right)$ defined by (4.3).

Theorem 5. If $b_{k} \geq b_{n}(k \geq n)$ and

$$
\theta=\frac{n \beta|b|[1+\gamma(p-q-1)] \delta(p, q)}{(n-p+\beta|b|)[1+\gamma(n-q-1)]\left[\frac{p+\lambda(n-p)}{p}\right]^{m} b_{n}}
$$

$(p>|b|)$, then

$$
C_{\gamma}^{q}(g(z) ; n, m, p, \lambda, \beta, b) \subset N_{n, p}^{\theta}\left(h^{(q)}\right) .
$$

Proof. Let $f \in C_{\gamma}^{q}(g(z) ; n, m, p, \lambda, \beta, b)$. Then, in view of the assertion (2.1) of Theorem 1, and the given condition that $b_{k} \geq b_{n}(k \geq n)$, we have

$$
\begin{gathered}
(n-p+\beta|b|)[1+\gamma(n-q-1)]\left[\frac{p+\lambda(n-p)}{p}\right]^{m} b_{n} \sum_{k=n}^{\infty} \delta(k, q) a_{k} \\
\leq \beta|b|[1+\gamma(p-q-1)] \delta(p, q)
\end{gathered}
$$

so that

$$
\sum_{k=n}^{\infty} \delta(k, q) a_{k} \leq \frac{\beta|b|[1+\gamma(p-q-1)] \delta(p, q)}{(n-p+\beta|b|)[1+\gamma(n-q-1)]\left[\frac{p+\lambda(n-p)}{p}\right]^{m} b_{n}}
$$


On the other hand, we also find from (2.1) and (4.7) that

$$
\begin{aligned}
\sum_{k=n}^{\infty} k \delta(k, q) a_{k} \leq & \frac{\beta|b|[1+\gamma(p-q-1)] \delta(p, q)}{[1+\gamma(n-q-1)]\left[\frac{p+\lambda(n-p)}{p}\right]^{m} b_{n}}+(p-\beta|b|) \sum_{k=n}^{\infty} \delta(k, q) a_{k} \\
\leq & \frac{\beta|b|[1+\gamma(p-q-1)] \delta(p, q)}{[1+\gamma(n-q-1)]\left[\frac{p+\lambda(n-p)}{p}\right]^{m} b_{n}} \\
& +\frac{(p-\beta|b|) \beta|b|[1+\gamma(p-q-1)] \delta(p, q)}{(n-p+\beta|b|)[1+\gamma(n-q-1)]\left[\frac{p+\lambda(n-p)}{p}\right]^{m} b_{n}}
\end{aligned}
$$

that is

$$
\sum_{k=n}^{\infty} \delta(k, q) k a_{k} \leq \frac{n \beta|b| \delta(p, q)[1+\gamma(p-q-1)]}{(n-p+\beta|b|)[1+\gamma(n-q-1)]\left[\frac{p+\lambda(n-p)}{p}\right]^{m} b_{n}}=\theta
$$

This evidently completes the proof of Theorem 5 .

Remark 1. (i) Taking $g(z)=\frac{z^{p}}{1-z}, b=\gamma, m=0$ and $\gamma=\lambda$ in Theorem 5, we obtain the result obtained by Altintaş et al. [2, Theorem 2];

(ii) Taking $g(z)=\frac{z^{p}}{1-z}, b=1, \beta=p-\alpha(0 \leq \alpha<p)$ and $\gamma=\lambda$ in Theorem 5 , we obtain the result obtained by Altintaş [1, Theorem 2].

Putting $g(z)=z^{p}+\sum_{k=n}^{\infty}\left[\frac{p+\ell+\zeta(k-p)}{p+\ell}\right]^{s} z^{k}\left(\ell, \zeta \geq 0 ; s \in \mathbb{N}_{0}\right)$ and $m=0$ in Theorem 5 , we obtain the following corollary.

Corollary 2. If $f(z) \in T_{p}(n)$ is in the class $C_{\gamma}^{q}(\zeta, \ell, s ; n, p, \beta, b)$, then

$$
C_{\gamma}^{q}(\zeta, \ell, s ; n, p, \beta, b) \subset N_{n, p}^{\theta}\left(h^{(q)}\right),
$$

where $h(z)$ is given by (4.2) and

$$
\theta=\frac{n \beta|b|[1+\gamma(p-q-1)] \delta(p, q)}{(n-p+\beta|b|)[1+\gamma(n-q-1)]}\left(\frac{p+\ell}{p+\ell+\zeta(n-p)}\right)^{s} .
$$

Putting $g(z)=z^{p}+\sum_{k=n}^{\infty}\left[\frac{p+\zeta(k-p)}{p}\right]^{s} z^{k}\left(\zeta \geq 0 ; s \in \mathbb{N}_{0}\right)$ and $m=0$ in Theorem 5 , we obtain the following corollary.

Corollary 3. If $f(z) \in T_{p}(n)$ is in the class $C_{\gamma}^{q}(\zeta, s ; n, p, \beta, b)$, then

$$
C_{\gamma}^{q}(\zeta, s ; n, p, \beta, b) \subset N_{n, p}^{\theta}\left(h^{(q)}\right),
$$

where $h(z)$ is given by (4.2) and

$$
\theta=\frac{n \beta|b|[1+\gamma(p-q-1)] \delta(p, q)}{(n-p+\beta|b|)[1+\gamma(n-q-1)]}\left(\frac{p}{p+\zeta(n-p)}\right)^{s} .
$$


Theorem 6. If

$$
\theta=\frac{n \beta|b|(p-q)}{[p-q+\gamma(n-p)]\left[\frac{p+\lambda(n-p)}{p}\right]^{m} b_{n}},
$$

then

$$
R_{\gamma}^{q}(g(z) ; n, m, p, \lambda, \beta, b) \subset N_{n, p}^{\theta}\left(h^{(q)}\right) .
$$

Proof. Let $f \in R_{\gamma}^{q}(g(z) ; n, m, p, \lambda, \beta, b)$. Then, in view of the assertion (2.3) of Theorem 2, we have

$$
\begin{aligned}
& \frac{[p-q+\gamma(n-p)]\left[\frac{p+\lambda(n-p)}{p}\right]^{m} b_{n}}{n} \sum_{k=n}^{\infty} \delta(k, q) k a_{k} \\
& \quad \leq \sum_{k=n}^{\infty}[p-q+\gamma(k-p)]\left[\frac{p+\lambda(k-p)}{p}\right]^{m} \delta(k, q) a_{k} b_{k} \\
& \quad \leq \beta|b|(p-q),
\end{aligned}
$$

so that

$$
\sum_{k=n}^{\infty} \delta(k, q) k a_{k} \leq \frac{n \beta|b|(p-q)}{[p-q+\gamma(n-p)]\left[\frac{p+\lambda(n-p)}{p}\right]^{m} b_{n}}=\theta
$$

which by means of the definition (4.1), establishes the inclusion (4.10) asserted by Theorem 6 .

Theorem 7. If $f(z) \in T_{p}(n)$ is in the class $H_{\gamma}^{q}(g(z) ; n, m, p, \lambda, \beta, b, \alpha)$, then

$$
H_{\gamma}^{q}(g(z) ; n, m, p, \lambda, \beta, b, \alpha) \subset N_{n, p}^{\theta}\left(f^{(q)}, k^{(q)}\right),
$$

where $k(z)$ is given by (1.13) and

$$
\theta=\frac{n \beta|b|[1+\gamma(p-q-1)][n+(p-q+\alpha)(p-q+\alpha+2)] \delta(p, q)}{(n-p+\beta|b|)[1+\gamma(n-q-1)]\left[\frac{p+\lambda(n-p)}{p}\right]^{m}(n-q+\alpha) b_{n}}
$$

Proof. Suppose that $f(z) \in H_{\gamma}^{q}(g(z) ; n, m, p, \lambda, \beta, b, \alpha)$. Then upon substituting from (3.5) into the following coefficient inequality

$$
\sum_{k=n}^{\infty} k \delta(k, q)\left|a_{k}-c_{k}\right| \leq \sum_{k=n}^{\infty} k \delta(k, q)\left|c_{k}\right|+\sum_{k=n}^{\infty} k \delta(k, q)\left|a_{k}\right|
$$

$\left(a_{k} ; c_{k} \geq 0\right)$, we readily obtain 


$$
\begin{aligned}
\sum_{k=n}^{\infty} k \delta(k, q)\left|a_{k}-c_{k}\right| \leq & \sum_{k=n}^{\infty} k \delta(k, q)\left|c_{k}\right| \\
& +\sum_{k=n}^{\infty} k \delta(k, q) \frac{(p-q+\alpha)(p-q+\alpha+1)}{(k-q+\alpha)(k-q+\alpha+1)}\left|c_{k}\right| .
\end{aligned}
$$

Now, since $k(z) \in C_{\gamma}^{q}(g(z) ; n, m, p, \lambda, \beta, b)$ the second assertion (4.8) yields

$$
k \delta(k, q) c_{k} \leq \frac{n \beta|b| \delta(p, q)[1+\gamma(p-q-1)]}{(n-p+\beta|b|)[1+\gamma(n-q-1)]\left[\frac{p+\lambda(n-p)}{p}\right]^{m} b_{n}} .
$$

Finally, by making use of (4.8) as well as (4.16) on the right-hand side of (4.15), we find that

$$
\begin{aligned}
\sum_{k=n+p}^{\infty} \delta(k, q) k\left|a_{k}-c_{k}\right| & \\
\leq & \frac{n \beta|b|[1+\gamma(p-q-1)] \delta(p, q)}{(n-p+\beta|b|)[1+\gamma(n-q-1)]\left[\frac{p+\lambda(n-p)}{p}\right]^{m} b_{n}} \\
& \quad \times\left(1+\sum_{k=n+p}^{\infty} \frac{(p-q+\alpha)(p-q+\alpha+1)}{(k-q+\alpha)(k-q+\alpha+1)}\right) \\
= & \frac{n \beta|b|[1+\gamma(p-q-1)][n+(p-q+\alpha)(p-q+\alpha+2)] \delta(p, q)}{(n-p+\beta|b|)[1+\gamma(n-q-1)](n+p-q+\alpha)\left[\frac{p+\lambda(n-p)}{p}\right]^{m} b_{n}} \\
= & \theta
\end{aligned}
$$

we conclude that $f \in N_{n, p}^{\theta}\left(f^{(q)}, k^{(q)}\right)$. This evidently completes the proof of Theorem 7 .

\section{Neighborhood for the classes $C_{\gamma}^{q, \zeta}(g(z) ; n, m, p, \lambda, \beta, b)$ and $R_{\gamma}^{q, \zeta}$} $(\boldsymbol{g}(\boldsymbol{z}) ; \boldsymbol{n}, \boldsymbol{m}, \boldsymbol{p}, \boldsymbol{\lambda}, \boldsymbol{\beta}, \boldsymbol{b})$. In this section we determine the neighborhood for the classes $C_{\gamma}^{q, \zeta}(g(z) ; n, m, p, \lambda, \beta, b)$ and $R_{\gamma}^{q, \zeta}(g(z) ; n, m, p, \lambda, \beta, b)$ which we define as follows. A function $f \in T_{p}(n)$ is said to be in the class $C_{\gamma}^{q, \zeta}(g(z) ; n$, $m, p, \lambda, \beta, b)$ if there exists a function $k \in C_{\gamma}^{q}(g(z) ; n, m, p, \lambda, \beta, b)$ such that

$$
\left|\frac{f(z)}{k(z)}-1\right|<p-\zeta
$$

$(z \in U ; 0 \leq \zeta<p)$ 
Theorem 8. If $k(z) \in C_{\gamma}^{q}(g(z) ; n, m, p, \lambda, \beta, b)$ and

$$
\zeta=p-\frac{\theta(n-p+\beta|b|)[1+\gamma(n-q-1)]\left[\frac{p+\lambda(n-p)}{p}\right]^{m} b_{n}}{n\left\{(n-p+\beta|b|)[1+\gamma(n-q-1)]\left[\frac{p+\lambda(n-p)}{p}\right]^{m} \delta(n, q) b_{n}-\beta|b|[1+\gamma(p-q-1)] \delta(p, q)\right\}},
$$

then

$$
N_{n, p}^{\theta}\left(k^{(q)}\right) \subset C_{\gamma}^{q, \zeta}(g(z) ; n, m, p, \lambda, \beta, b),
$$

where

$$
\begin{aligned}
\theta \leq n p[\delta(n, q) & -\beta|b|[1+\gamma(p-q-1)] \delta(p, q) \\
& \left.\times\left\{(n-p+\beta|b|)[1+\gamma(n-q-1)]\left[\frac{p+\lambda(n-p)}{p}\right]^{m} b_{n}\right\}^{-1}\right] .
\end{aligned}
$$

Proof. Suppose that $f \in N_{n, p}^{\theta}\left(k^{(q)}\right)$, then we find from the definition (4.1) that

$$
\sum_{k=n}^{\infty} \delta(k, q) k\left|a_{k}-c_{k}\right| \leq \theta
$$

which implies the coefficient inequality

$$
\sum_{k=n}^{\infty}\left|a_{k}-c_{k}\right| \leq \frac{\theta}{n \delta(n, q)}
$$

$\left(p>q ; n, p \in \mathbb{N}, q \in \mathbb{N}_{0}\right)$. Next, since $k(z) \in C_{\gamma}^{q}(g(z) ; n, m, p, \lambda, \beta, b)$, we have

$$
\sum_{k=n}^{\infty} c_{k} \leq \frac{\beta|b|[1+\gamma(p-q-1)] \delta(p, q)}{(n-p+\beta|b|)[1+\gamma(n-q-1)]\left[\frac{p+\lambda(n-p)}{p}\right]^{m} \delta(n, q) b_{n}},
$$

so that

$$
\begin{aligned}
\left|\frac{f(z)}{k(z)}-1\right| & \leq \frac{\sum_{k=n}^{\infty}\left|a_{k}-c_{k}\right|}{1-\sum_{k=n}^{\infty}\left|c_{k}\right|} \\
& \leq \frac{\frac{\theta}{n \delta(n, q)}}{1-\frac{\beta|b|[1+\gamma(p-q-1)] \delta(p, q)}{(n-p+\beta|b|)[1+\gamma(n-q-1)]\left[\frac{p+\lambda(n-p)}{p}\right]^{m} \delta(n, q) b_{n}}} \\
& =\frac{\theta(n-p+\beta|b|)[1+\gamma(n-q-1)]\left[\frac{p+\lambda(n-p)}{p}\right]^{m} b_{n}}{n\left\{(n-p+\beta|b|)[1+\gamma(n-q-1)]\left[\frac{p+\lambda(n-p)}{p}\right]^{m} \delta(n, q) b_{n}-\beta|b| \delta(p, q)[1+\gamma(p-q-1)]\right\}} \\
& =p-\zeta,
\end{aligned}
$$


because by the assumption

$$
\zeta=p-\frac{\theta(n-p+\beta|b|)[1+\gamma(n-q-1)]\left[\frac{p+\lambda(n-p)}{p}\right]^{m} b_{n}}{n\left\{(n-p+\beta|b|)[1+\gamma(n-q-1)]\left[\frac{p+\lambda(n-p)}{p}\right]^{m} \delta(n, q) b_{n}-\beta|b| \delta(p, q)[1+\gamma(p-q-1)]\right\}} .
$$

This implies that $f \in C_{\gamma}^{q, \zeta}(g(z) ; n, m, p, \lambda, \beta, b)$.

Similarly, we can prove the following theorem.

Theorem 9. If $k(z) \in R_{\gamma}^{q}(g(z) ; n, m, p, \lambda, \beta, b)$ and

$$
\zeta=p-\frac{\theta[p-q+\gamma(n-p)]\left[\frac{p+\lambda(n-p)}{p}\right]^{m} b_{n}}{n\left\{[p-q+\gamma(n-p)]\left[\frac{p+\lambda(n-p)}{p}\right]^{m} \delta(n, q) b_{n}-\beta|b|(p-q)\right\}},
$$

then

$$
N_{n, p}^{\theta}\left(k^{(q)}\right) \subset R_{\gamma}^{q, \zeta}(g(z) ; n, m, p, \lambda, \beta, b),
$$

where

$$
\theta \leq n p\left[\delta(n, q)-\beta|b|(p-q)\left\{[p-q+\gamma(n-p)]\left[\frac{p+\lambda(n-p)}{p}\right]^{m} b_{n}\right\}^{-1}\right] .
$$

\section{REFERENCES}

[1] Altintaş, O., Neighborhoods of certain p-valently analytic functions with negative coefficients, Appl. Math. Comput. 187 (2007), 47-53.

[2] Altintaş, O., Irmak, H. and Srivastava, H. M., Neighborhoods for certain subclasses of multivalently analytic functions defined by using a differential operator, Comput. Math. Appl. 55 (2008), 331-338.

[3] Altintaş, O., Özkan, Ö. and Srivastava, H. M., Neighborhoods of a certain family of multivalent functions with negative coefficient, Comput. Math. Appl. 47 (2004), $1667-1672$.

[4] Aouf, M. K., Inclusion and neighborhood properties for certain subclasses of analytic functions associated with convolution structure, J. Austral. Math. Anal. Appl. 6, no. 2 (2009), Art. 4, 1-10.

[5] Aouf, M. K., Mostafa, A. O., On a subclass of n-p-valent prestarlike functions, Comput. Math. Appl. 55 (2008), 851-861.

[6] Aouf, M. K., Seoudy, T. M., On differential sandwich theorems of analytic functions defined by certain linear operator, Ann. Univ. Marie Curie-Skłodowska Sect. A, 64 (2) (2010), 1-14.

[7] Cătaş, A., On certain classes of p-valent functions defined by multiplier transformations, Proceedings of the International Symposium on Geometric Function Theory and Applications: GFTA 2007 Proceedings (İstanbul, Turkey; 20-24 August 2007) (S. Owa and Y. Polatoglu, Editors), pp. 241-250, TC İstanbul Kültűr University Publications, Vol. 91, TC İstanbul Kültür University, İstanbul, Turkey, 2008.

[8] El-Ashwah, R. M., Aouf, M. K., Inclusion and neighborhood properties of some analytic p-valent functions, General Math. 18, no. 2 (2010), 173-184.

[9] Frasin, B. A., Neighborhoods of certain multivalent analytic functions with negative coefficients, Appl. Math. Comput. 193, no. 1 (2007), 1-6. 
[10] Goodman, A. W., Univalent functions and non-analytic curves, Proc. Amer. Math. Soc. 8 (1957), 598-601.

[11] Kamali, M., Orhan, H., On a subclass of certain starlike functions with negative coefficients, Bull. Korean Math. Soc. 41, no. 1 (2004), 53-71.

[12] Mahzoon, H., Latha, S., Neighborhoods of multivalent functions, Internat. J. Math. Analysis, 3, no. 30 (2009), 1501-1507.

[13] Orhan, H., Kiziltunc, H., A generalization on subfamily of p-valent functions with negative coefficients, Appl. Math. Comput., 155 (1004), 521-530.

[14] Prajapat, J. K., Raina, R. K. and Srivastava, H. M., Inclusion and neighborhood properties of certain classes of multivalently analytic functions associated with convolution structure, JIPAM. J. Inequal. Pure Appl. Math. 8, no. 1 (2007), Article 7, 8 pp. (electronic).

[15] Raina, R. K., Srivastava, H. M., Inclusion and neighborhood properties of some analytic and multivalent functions, J. Inequal. Pure Appl. Math. 7, no. 1 (2006), 1-6.

[16] Ruscheweyh, St., Neighborhoods of univalent functions, Proc. Amer. Math. Soc. 81 (1981), 521-527.

[17] Srivastava, H. M., Orhan, H., Coefficient inequalities and inclusion relations for some families of analytic and multivalent functions, Applied Math. Letters, 20, no. 6 (2007), 686-691.

[18] Srivastava, H. M., Suchithra, K., Stephen, B. A. and Sivasubramanian, S., Inclusion and neighborhood properties of certain subclasses of analytic and multivalent functions of complex order, JIPAM. J. Inequal. Pure Appl. Math. 7, no. 5 (2006), Article 191, 8 pp. (electronic).

R. M. El-Ashwah

Department of Mathematics

Faculty of Science at Damietta

University of Mansoura

New Damietta 34517

Egypt

e-mail: r_elashwah@yahoo.com

\section{S. M. El-Deeb}

Department of Mathematics Faculty of Science at Damietta

University of Mansoura

New Damietta 34517

Egypt

e-mail: shezaeldeeb@yahoo.com

Received March 2, 2010
M. K. Aouf

Department of Mathematics

Faculty of Science

University of Mansoura

Mansoura 33516

Egypt

e-mail: mkaouf 127@yahoo.com 